\title{
Choke-mode damped structure design for the Compact Linear Collider main linac
}

\author{
Hao Zha, ${ }^{1}$ Jiaru Shi, ${ }^{1,2, *}$ Huaibi Chen, ${ }^{1, \dagger}$ Alexej Grudiev, ${ }^{2}$ Walter Wuensch, ${ }^{2}$ Chuanxiang Tang, ${ }^{1}$ and Wenhui Huang ${ }^{1}$ \\ ${ }^{1}$ Tsinghua University, Beijing CN-100084, People's Republic of China \\ ${ }^{2}$ CERN, Geneva CH-1211, Switzerland
}

(Received 24 April 2012; published 10 December 2012)

\begin{abstract}
Choke-mode damped structures are being studied as an alternative design to waveguide damped structures for the main linac of the Compact Linear Collider (CLIC). Choke-mode structures have the potential for lower pulsed temperature rise and simpler and less expensive fabrication. An equivalent circuit model based on transmission line theory for higher-order-mode damping is presented. Using this model, a new choke geometry is proposed and the wakefield performance is verified using GDFIDL. This structure has a comparable wakefield damping effect to the baseline design which uses waveguide damping. A prototype structure with the same iris dimensions and accelerating gradient as the nominal CLIC design, but with the new choke geometry, has been designed for high-power tests.
\end{abstract}

DOI: 10.1103/PhysRevSTAB.15.122003

PACS numbers: 29.20.Ej, 41.75.Ht

\section{INTRODUCTION}

The CLIC-G design with waveguide damping is the baseline accelerating structure design for the Compact Linear Collider (CLIC) main linac [1]. It operates at $11.994 \mathrm{GHz}$ in the $2 \pi / 3$ mode at an accelerating gradient of $100 \mathrm{MV} / \mathrm{m}$. It has beam aperture with an average radius of $2.75 \mathrm{~mm}$, and a corresponding average group velocity of $\sim 1.3 \%$ c. Multibunch beam dynamics in the CLIC main linac have been studied in [2] and give a wakefield suppression constraint to avoid beam instability: the transverse kick applied by each bunch to the next following bunch should be below $6.6 \mathrm{~V} /(\mathrm{m} \mathrm{mm} \mathrm{pC})$. The CLIC-G waveguide damping cells suppress the transverse wakefield to a level of about $5 \mathrm{~V} /(\mathrm{m} \mathrm{mm} \mathrm{pC})$ at the position of the second bunch, which meets the beam dynamics requirements.

The choke-mode accelerating structure was first proposed by Tsumoru Shintake [3], as shown in Fig. 1. The principle of operation is that the choke reflects the accelerating mode, but unwanted higher order modes (HOMs) can pass the choke to be absorbed in the load.

The choke-mode design has several potential advantages to other types of heavy damped structures: (i) it has lower surface magnetic field compared to waveguide damped structures, which results in lower pulsed temperature rise; (ii) there are no bonding joints of the disks in high magnetic field; (iii) the choke structure is axially symmetric so can be manufactured by turning, which potentially greatly reduces the total manufacturing cost.

\footnotetext{
*shij@mail.tsinghua.edu.cn

${ }^{\dagger}$ chenhb@mail.tsinghua.edu.cn
}

Published by the American Physical Society under the terms of the Creative Commons Attribution 3.0 License. Further distribution of this work must maintain attribution to the author(s) and the published article's title, journal citation, and DOI.
Given these benefits, the choke-mode structures have been studied as an alternative design for the CLIC main linacs in a collaboration between CERN and Tsinghua University. Other alternative designs include detuned damping structures [4]; iris slot structures [5] are also being studied.

The choke design does however have certain challenges [3]: (i) the larger volume and surface area make the chokemode cell a lower rf-beam efficiency; (ii) modes at a frequency near odd multiple of the accelerating frequency are reflected by choke and are consequently not well damped, which may include modes with high $(R / Q)_{\perp}$; (iii) the high-gradient performance of $\mathrm{X}$-band chokemode structures is still not clear.

We introduce a transmission line model for choke-mode structures in the next section. Section III discusses the wakefield damping of an ordinary choke-mode structure. A new choke geometry design and its wakefield simulation is presented in Sec. IV. The final section discusses rf parameters of this new choke design.

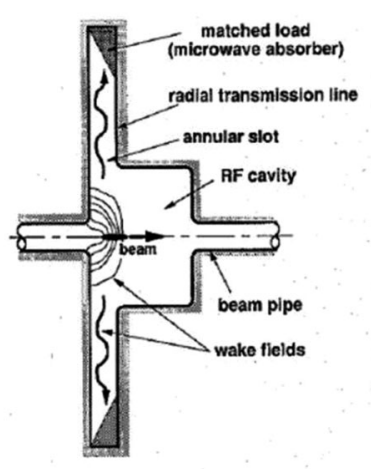

(a) Radial Line Damper

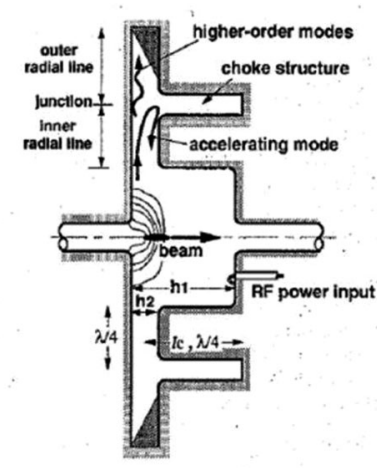

(b) Choke Mode Cavity
FIG. 1. The choke-mode cavity [3] 


\section{TRANSMISSION LINE MODEL FOR CHOKE MODE}

The rf properties of choke structures can be modeled with a combination of four uniform lossless transmission lines as shown in Fig. 2. The transmission lines have different characteristic impedances which mainly depend on the gap height [3]. Since the choke and outer radial line are in series, the impedance at the junction plane is the sum of the choke and the outer radial line.

The outer radial line is terminated by an absorber and can be modeled as perfectly matched load with impedance equal to 1 . The top of the choke is a short circuit. The impedance at the open end of the choke, reference plane A, is pure imaginary in the case that resistive losses are not considered. For the coaxial choke, $\varphi_{3}=k c_{3}$, where $c_{3}$ is the length of the choke, $k$ is the wave number. The impedance of choke becomes $z_{3}=j \tan \left(k c_{3}\right)$ and the total impedance at reference plane $\mathrm{T}$ is $z_{2}^{\prime}=1+j \tan \left(k c_{3}\right)$.

For the accelerating mode, $\varphi_{3}=\pi / 2$, so the impedance is infinite and the mode is fully reflected by the choke. For other modes the choke impedance is finite and the mode will be partially reflected, and the transmitted part will be damped by the absorber in the radial line. This can be visualized with a Smith chart, as shown in Fig. 3. At the top of the choke, the impedance of all modes is zero and is

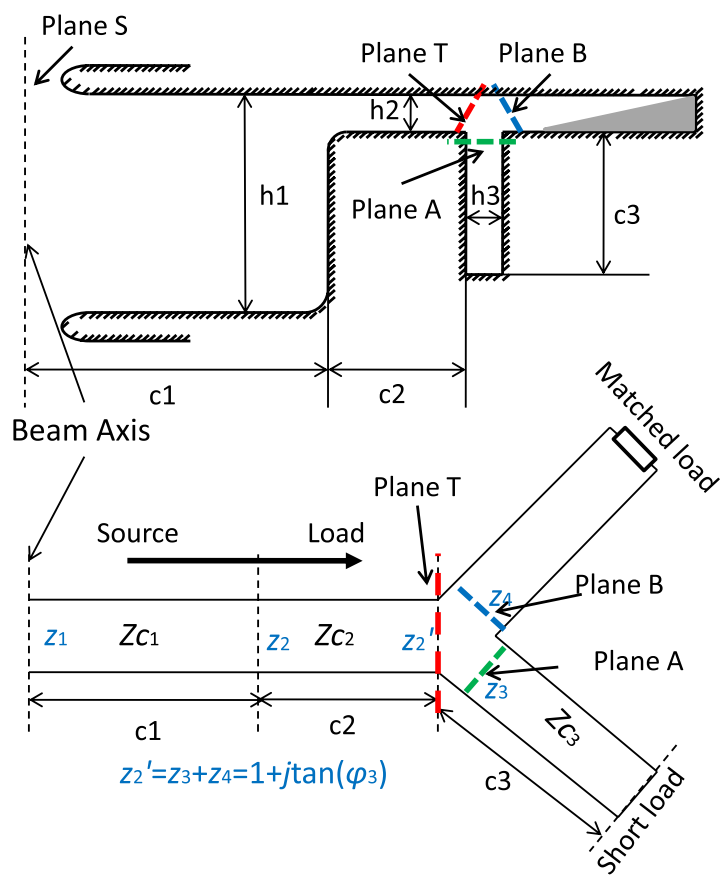

FIG. 2. The transmission line model for choke-mode cavity. The subscripts $i=1,2,3$, and 4 represent variables of the main cavity, the inner radial line, the choke structure, and the outer radial line, respectively. $c_{i}$ is the size (radius) of each branch; $Z_{c i}$ is the characteristic impedance; $\varphi_{i}$ is the phase advance for a traveling wave through each branch. If we consider the beam axis (reference plane $\mathrm{S}$ ) as source, $z_{i}$ is the equivalent impedance at each reference plane.
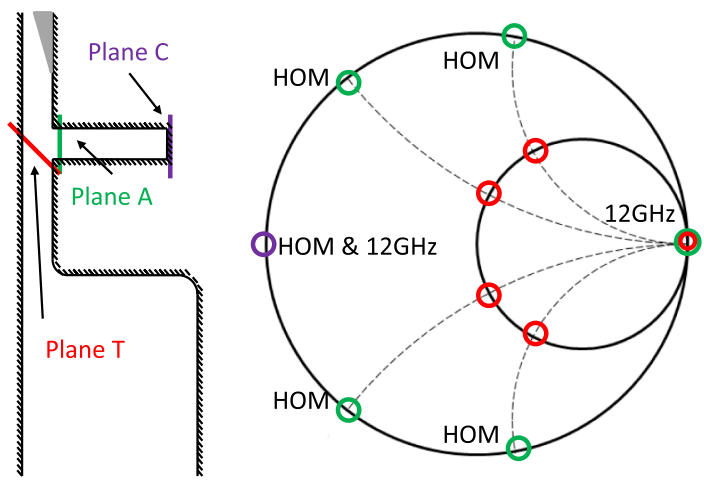

FIG. 3. Smith chart explanation. Blue: Impedance at the top surface of choke (reference plane C). Red: Impedance at the termination of choke (reference plane A). Green: Impedance at the junction plane of choke and radial line (reference plane $\mathrm{T}$ ).

shown as a short point in a Smith chart. Since the choke is a uniform transmission line, these points will move along on the pure reactance circle in a Smith chart. At the open end of the choke, reference plane A, the accelerating mode becomes an open point in a Smith chart, while other modes are distributed around the pure reactance circle. At the junction plane, reference $\mathrm{T}$, the total impedance is 1 from the absorber plus the reactance from the choke, so all modes are distributed on a constant resistance circle. The accelerating mode is still at the open point and is consequently fully reflected by the choke. The higher order modes are shown as other points in a Smith chart so the resulting reflection is less than 1 .

The impedance $z_{1}$ at the beam axis, show in Fig. 2 as reference plane $\mathrm{S}$, determines the voltage in the transmission line model. If the curve of the impedance as a function of frequency, $z_{1}(f)$, is known, each peak in this curve corresponds to a mode. The center frequency of a particular peak is the resonant frequency of this mode and the width of the peak indicates the external $Q$ factor.

We have $\varphi_{i}=\pi / 2$ (see Fig. 2) for each branch for the accelerating mode in an ideal choke, where $\varphi$ is the phase shift in each branch. Assuming that the real phase shift is $\varphi_{i}=\Delta \varphi_{i}+\pi / 2$, here the $\Delta \varphi_{i}$ is given by the offset of dimensions and frequency change. The impedance $z_{1}$ can be derived as a function of $\Delta \varphi_{i}$ as follows:

$$
\begin{aligned}
& z_{3}=j \tan \left(\varphi_{3}\right)=j \tan \left(\Delta \varphi_{3}+\pi / 2\right) \approx j / \Delta \varphi_{3}, \\
& z_{2}^{\prime}=\frac{z_{3}}{M}+z_{4}=1+\frac{j}{M \Delta \varphi_{3}}, \\
& z_{2}=\frac{z_{2}^{\prime}+j \tan \left(\varphi_{2}\right)}{1+j \tan \left(\varphi_{2}\right) z_{2}^{\prime}} \approx \frac{\Delta \varphi_{2} z_{2}^{\prime}+j}{\Delta \varphi_{2}+j z_{2}^{\prime}} \\
& z_{1}=\frac{z_{2} / N+j \tan \left(\varphi_{1}\right)}{1+j \tan \left(\varphi_{1}\right) z_{2} / N} \approx \frac{z_{2} \Delta \varphi_{1}+j N}{N \Delta \varphi_{1}+j z_{2}}, \\
& N=Z c_{1} / Z c_{2}, \quad M=Z c_{2} / Z c_{3},
\end{aligned}
$$

where $\varphi_{1}, \varphi_{2}$, and $\varphi_{3}$ are the phase shifts of main cavity, inner radial line, and the choke. $Z c_{i}$ is the characteristic 
impedance of each branch, shown in Fig. 2, and is proportional to the gap size of each branch. Then we have

$$
N=h_{1} / h_{2}, \quad M=h_{2} / h_{3} .
$$

Here $h_{1}, h_{2}$, and $h_{3}$ are the gap sizes of main cavity, inner radial line, and choke. Because of the difference between the characteristic impedances, $z_{3}$ and $z_{2}$ will be divided by $M$ and $N$. From Eq. (1), the approximate expression on $z_{1}$ is

$$
z_{1} \approx \frac{j N}{N \Delta \varphi_{1}+\Delta \varphi_{2}+M \Delta \varphi_{3}+j\left(M \Delta \varphi_{3}\right)^{2}} .
$$

For the accelerating mode of choke-mode cavity,

$$
\begin{gathered}
\frac{\partial \varphi_{i}}{\partial f} \approx \frac{k c_{i}}{f_{0}}, \frac{\partial \varphi_{i}}{\partial c_{i}} \approx k \Rightarrow \\
\Delta \varphi_{i}=k c_{i} \frac{\Delta f}{f_{0}}+k c_{i} \frac{\Delta c_{i}}{c_{i}}=\rho_{i}\left(\frac{\Delta f}{f_{0}}+\frac{\Delta c_{i}}{c_{i}}\right),
\end{gathered}
$$

where $c_{i}$ is the ideal size (or radius), $f_{0}$ is the ideal resonant frequency.

The electromagnetic field solutions in cylindrical coordinates give $\rho_{i}=k c_{i}(i=1,2,3)$, which are roots of the Bessel functions: $\rho_{1} \approx 2.405$ is the first root of $J_{0}(x)$, and $\rho_{2} \approx 3.832-\rho_{1}$, making $\left(\rho_{1}+\rho_{2}\right)$ the first root of $J_{0}^{\prime}(x)$. For the coaxial choke we have $\rho_{3}=\pi / 2$.

From Eq. (3), $z_{1}$ reaches its maximum when $N \Delta \varphi_{1}+$ $\Delta \varphi_{2}+M \Delta \varphi_{3}=0$. The frequency which fits this equation is the real resonant frequency $\left(f_{0}+\Delta f\right)$. When $N \Delta \varphi_{1}+$ $\Delta \varphi_{2}+M \Delta \varphi_{3}=\left(M \Delta \varphi_{3}\right)^{2}$ the impedance $z_{1}$ will be $1 / \sqrt{2}$ of its maximum value. This indicates the external $Q$ as

$$
\begin{aligned}
& \left.\left(N \Delta \varphi_{1}+\Delta \varphi_{2}+M \Delta \varphi_{3}\right)\right|_{f=f_{0}+\Delta f}=0, \\
& \left.\left(N \Delta \varphi_{1}+\Delta \varphi_{2}+M \Delta \varphi_{3}\right)\right|_{f=f_{0}+\Delta f \pm \delta f}=\left(M \Delta \varphi_{3}\right)^{2} \\
& \Rightarrow \\
& \frac{\Delta f}{f_{0}} \approx-\frac{1}{N^{*}+1+M^{*}}\left(N^{*} \frac{\Delta c_{1}}{c_{1}}+\frac{\Delta c_{2}}{c_{2}}+M^{*} \frac{\Delta c_{3}}{c_{3}}\right), \\
& Q_{\mathrm{ext}} \approx \frac{\left(N^{*}+1+M^{*}\right)^{3}}{2 \rho_{2}\left[M^{*}\left(N^{*} \frac{\Delta c_{1}}{c_{1}}+\frac{\Delta c_{2}}{c_{2}}-\left(N^{*}+1\right) \frac{\Delta c_{3}}{c_{3}}\right)\right]^{2}},
\end{aligned}
$$

where $\quad \delta f=f_{0} / 2 Q_{\text {ext }}, \quad$ and $\quad N^{*}=N \rho_{1} / \rho_{2}, \quad M^{*}=$ $M \rho_{3} / \rho_{2}$. We call Eq. (5) the tuning formula and it will be used for simulations of rf design. In the design of a choke-mode cavity, the radii of the choke and the inner radial line are adjusted to keep the frequency constant and the external $Q$ factor at a high level. Given initial values for the radius of the choke and inner radial line, the resonant frequency and external $Q$ factor can be obtained by simulation. Then the adjustments of choke and radial line are calculated using Eq. (6) shown below, derived from Eq. (5) when $c_{1}$ is constant. With this formula, the proper radii of the choke and inner radial line are obtained within several iterations:
TABLE I. Change of frequency and $Q$ factor in different machining accuracies, $Q_{0} / Q_{\text {ext }}=-\Delta Q / Q$ is the $Q$ decrease, with the assumption that $Q_{0} \approx 5000$.

\begin{tabular}{lcrrr}
\hline \hline Parameters & \multicolumn{2}{c}{$\Delta f(\mathrm{MHz})$} & \multicolumn{2}{c}{$Q_{0} / Q_{\text {ext }}$} \\
Accuracy & $5 \mu \mathrm{m}$ & $10 \mu \mathrm{m}$ & $5 \mu \mathrm{m}$ & $10 \mu \mathrm{m}$ \\
\hline Main cavity & 5.1 & 10.2 & $0.031 \%$ & $0.122 \%$ \\
Inner radial line & 0.9 & 1.8 & $0.001 \%$ & $0.004 \%$ \\
Choke & 0.9 & 1.8 & $0.078 \%$ & $0.311 \%$ \\
Worst case & 6.9 & 13.8 & $0.235 \%$ & $0.941 \%$ \\
\hline \hline
\end{tabular}

$$
\begin{aligned}
\frac{\Delta c_{3}}{c_{3}} & \approx-\frac{\Delta f}{f_{0}} \pm \sqrt{\frac{N^{*}+1+M^{*}}{2 \rho_{2} Q_{\mathrm{ext}} M^{* 2}}}, \\
\frac{\Delta c_{2}}{c_{2}} & \approx-\left(N^{*}+1+M^{*}\right) \frac{\Delta f}{f_{0}}-M^{*} \frac{\Delta c_{3}}{c_{3}} .
\end{aligned}
$$

The machining tolerance of the choke-mode cavity can be estimated by the tuning formula [Eq. (5)]. For a chokemode cavity with a $1.5-\mathrm{mm}$ gap radial choke working at $11.994 \mathrm{GHz}$ with $2 \pi / 3$ mode, the dimensions $c_{1}, c_{2}$, and $c_{3}$ of this cell are approximately $9.5,5.5$, and $6.5 \mathrm{~mm}$, respectively. The frequency and $Q$-factor changes are calculated assuming machining errors of 5 and $10 \mu \mathrm{m}$. The change of $Q$ factor is calculated by adding external losses $\left(Q_{\text {ext }}\right)$ to the wall loss $\left(Q_{0}\right)$ :

$$
\frac{1}{Q}=\frac{1}{Q_{0}}+\frac{1}{Q_{\mathrm{ext}}}, \quad \frac{\Delta Q}{Q}=\frac{Q-Q_{0}}{Q}=-\frac{Q_{0}}{Q_{\mathrm{ext}}} .
$$

The results are listed in Table I, by giving the dimensional error to each branch separately, and a worst case is also estimated to indicate the maximum possible change at a certain machining accuracy. According to this table, the $10 \mu \mathrm{m}$ tolerance for inner radial line and choke are acceptable, but for the main cavity reaching a machining accuracy of $5 \mu \mathrm{m}$ is necessary to avoid heavy tuning to the structure [6].

\section{WAKEFIELD DAMPING IN ORDINARY CHOKE STRUCTURES}

The original design of the choke-mode structure used a coaxial choke with the length of $\lambda / 4$ [3]. However, the longitudinal length of the cells of CLIC main linacs is $\lambda / 3$ ( $2 \pi / 3$ mode) which is only $2 \mathrm{~mm}$ longer than the coaxial choke. The radial line of the choke also occupies some space so the remaining material is very limited. This point is mechanically weak and is a bottleneck for dissipation of the heat generated in the cavity [7].

A radial choke design, shown in Fig. 4, gives enough space for conducting the surface heating and has better mechanic rigidity. Choke design with a wider gap will also be possible in this configuration.

Once the parameters of the choke are designed for the fundamental mode, the corresponding transverse wakefield damping must be determined. This has been done using 


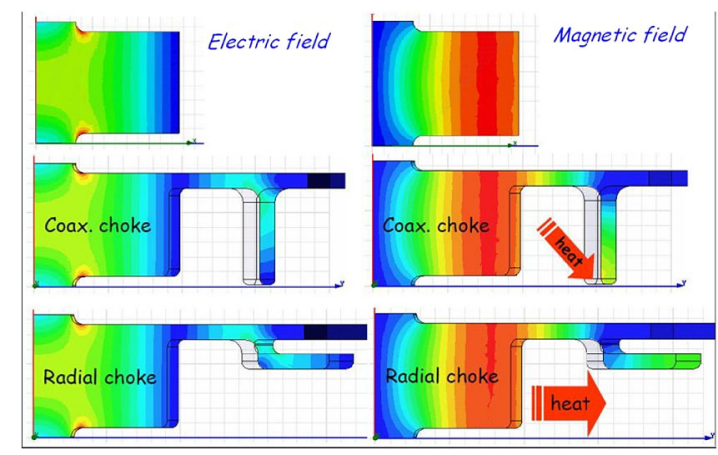

FIG. 4. Radial choke [7].

GDFIDL [8]. The starting point was the transverse wakefield in a structure with 24 tapered cells similar to the nominal CLIC-G design, but with this radial choke. We have named this structure series as "CDS," and the first configuration is called "CDS-A." The iris dimensions are taken directly from the CLIC-G design. The iris radii of the first cell and the last cell are 2.35 and $3.15 \mathrm{~mm}$, respectively. The structure has a radial choke with 1-mm gap. The absorber in the outer radial line is modeled by perfectly matched layers in these simulations. The simulation results are shown in Fig. 5. In this CDS-A structure, the wakefield is damped more slowly than in waveguide damped structures. At the point of the second bunch, the transverse wake potential of CDS-A is about $20-30 \mathrm{~V} /(\mathrm{m} \mathrm{mm} \mathrm{pC})$, while that of the waveguide damping design is $5 \mathrm{~V} /(\mathrm{m} \mathrm{mm} \mathrm{pC})$.

Figure 6 shows the impedance spectrum of the wakefield corresponding. There is a high peak near $29 \mathrm{GHz}$, which is caused by a weakly damped mode with a $Q$ factor of about 100. This mode gives the main contribution to the total transverse wakefield potential at the second bunch. There are also two modes near $17 \mathrm{GHz}$ which are the first-passband dipole modes. They have a lower $Q$ and

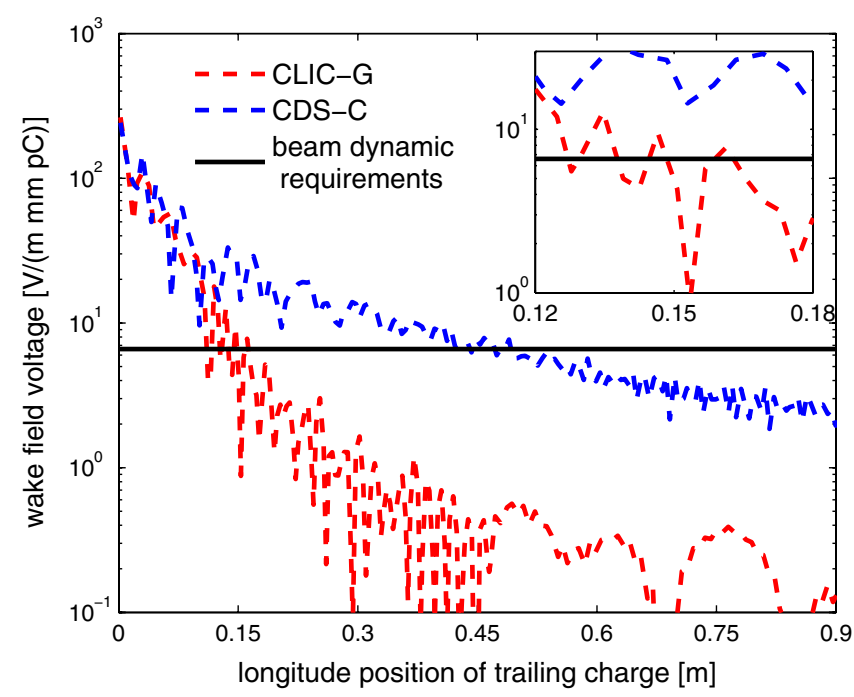

FIG. 5. Transverse wakefield potential of CDS-A (24 tapered cells with ordinary 1-mm gap radial choke).

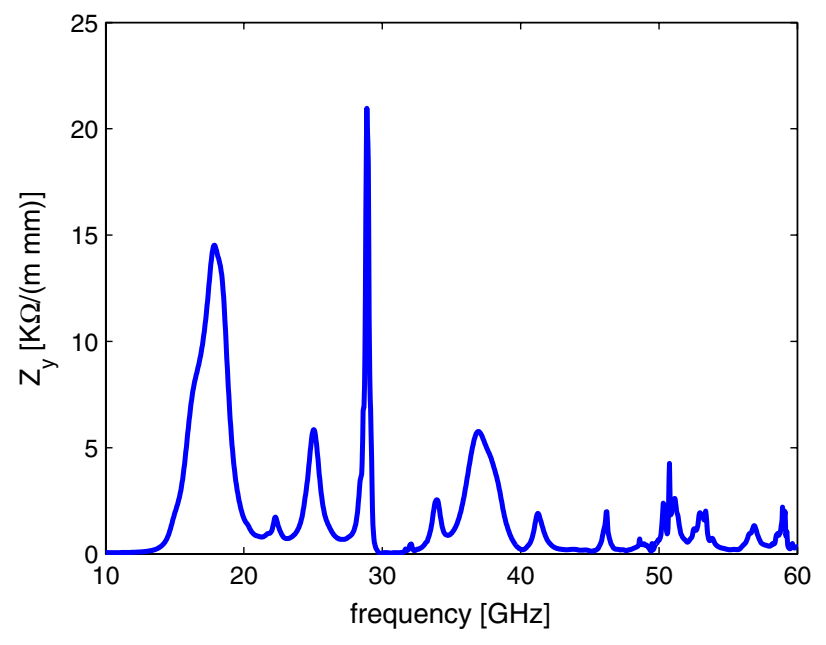

FIG. 6. Wakefield impedance spectrum of CDS-A.

amplitude. The mode near $16 \mathrm{GHz}$ has extra-lower amplitude which has been submerged in the curve of wakefield impedance. Despite the lower peak amplitude of the two modes, they have very large $(R / Q)_{\perp}$ so they may also make a large contribution to the total transverse wakefield. Other modes at higher frequency have much lower $(R / Q)_{\perp}$, therefore they do not have a big transverse kick to the beam even if they are modestly damped.

In order to quickly determine the absorption of higher order modes by the choke structure, the simple model shown in Fig. 7 can be used. It is similar to the model in [9]. The radial line with a choke is connected to a coaxial line, which can be defined as a waveguide port during simulation. The outer layer of the radial line is filled with absorber. We simulate the reflection at the port of the coaxial line, and can even measure it if a prototype structure is built. After calibration, the reflection seen from the choke $\left(S_{11}\right)$ can be obtained.

Figure 8 shows the results of HFSS simulation [10] for this structure. The zero absorption points in the curve indicate that modes at these frequencies are fully reflected by the choke. The first fully reflected frequency must correspond to the accelerating mode. The second fully reflected frequency is about $29.3 \mathrm{GHz}$ and there is a dipole mode near this frequency at the same time. This is the

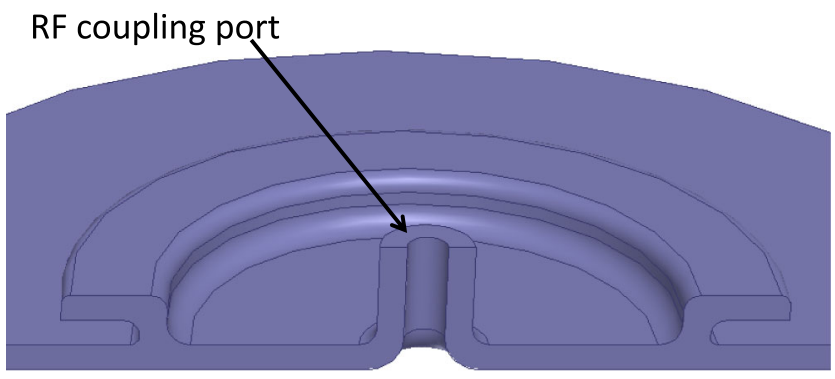

FIG. 7. Structures to test the reflection at choke. 


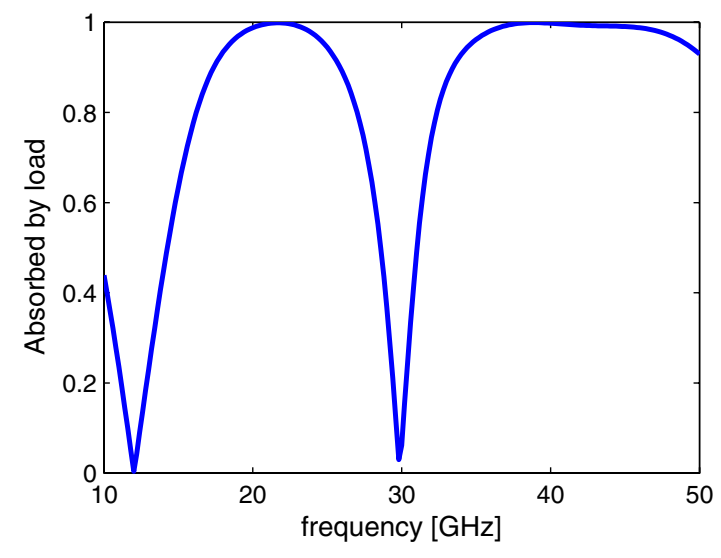

FIG. 8. Absorption curve of choke. Absorption in the load is given by $\sqrt{1-S_{11}^{2}}$.

reason for the high peak near $29 \mathrm{GHz}$. To avoid this serious problem, the second fully reflected frequency must be moved to a higher frequency which is far away from all the dipole modes with high $(R / Q)_{\perp}$.

\section{NEW CHOKE GEOMETRY DESIGN}

In order to avoid this higher order reflection from the choke, the new choke geometry shown in Fig. 9 is proposed. Compared with the ordinary choke design, there are three new features: (i) the new choke has two sections with different gaps and the gap of the choke can be different from the radial line; (ii) there is a step in outer radial line; (iii) the radii of the main cell and the inner radial line are adjusted to detune dipole modes. These new features will be discussed in the following subsections.

\section{A. Choke with two sections}

As shown in Fig. 10, the gaps of two sections are different. Consequently, the joint plane becomes an impedance transformer. This type of choke geometry was first introduced in [11]. Assuming that the two sections are coaxial lines with lengths $L_{\mathrm{a}}$ and $L_{\mathrm{b}}$ and the ratio of gap size is $K$, we can calculate the reactance of the choke:

$$
\begin{aligned}
& z_{\mathrm{b}}=j \tan \left(k L_{\mathrm{b}}\right), \quad z_{\mathrm{a}}=K z_{\mathrm{b}}=j K \tan \left(k L_{\mathrm{b}}\right), \\
& z_{3}=\frac{z_{\mathrm{a}}+j \tan \left(k L_{\mathrm{a}}\right)}{j z_{\mathrm{a}} \tan \left(k L_{\mathrm{b}}\right)+1}=j \frac{\tan \left(k L_{\mathrm{a}}\right)+K \tan \left(k L_{\mathrm{b}}\right)}{1-K \tan \left(k L_{\mathrm{a}}\right) \tan \left(k L_{\mathrm{b}}\right)},
\end{aligned}
$$

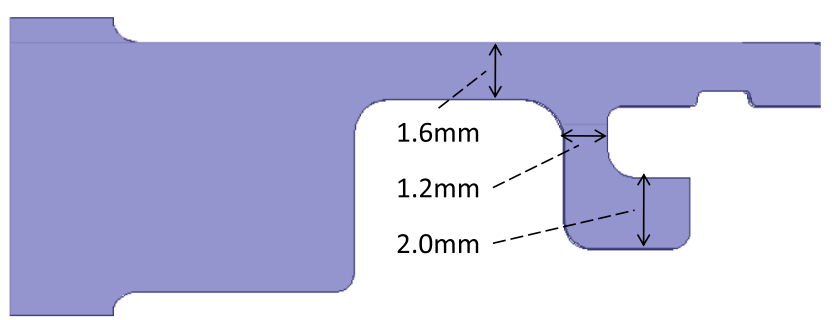

FIG. 9. New choke geometry.

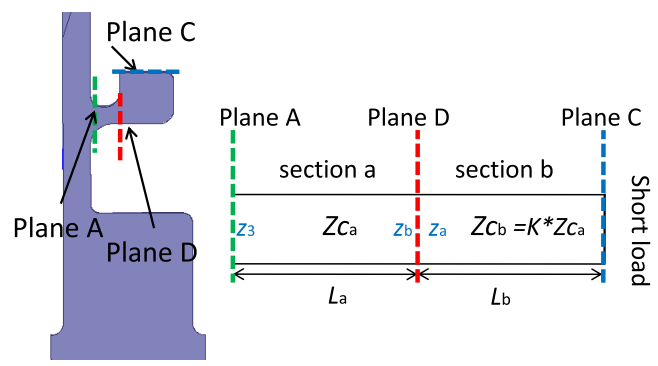

FIG. 10. Choke with two sections.

where $k$ is the wave number. The impedance from choke $z_{3}$ will be infinite when this wave number satisfies the equation below:

$$
\tan \left(k L_{\mathrm{a}}\right) \tan \left(k L_{\mathrm{b}}\right)=\frac{1}{K} .
$$

The first root of this equation is the wave number of the accelerating mode. The second root corresponds to the second fully reflected frequency. When the two sections have the same length $L_{\mathrm{a}}=L_{\mathrm{b}}$, the second fully reflected frequency is given by

$$
\frac{f_{2}}{f_{1}}=\frac{k_{2}}{k_{1}}=\frac{\pi}{\tan ^{-1}\left(K^{-1 / 2}\right)}-1,
$$

where $f_{2}$ is the second fully reflected frequency, $f_{1}$ is the frequency of the accelerating mode, and $k_{i}$ is the $i$ th root of Eq. (8). For the ordinary coaxial choke, $K=1$, consequently the second fully reflected frequency is about 3 times the accelerating mode. When $K>1$, the frequency of the second full reflection will be higher.

For the new choke geometry design shown in Fig. 9, the gaps of two sections are 1.2 and $2.0 \mathrm{~mm}$. The second fully reflected frequency is near $45 \mathrm{GHz}$ according to Eq. (9). A similar model as Fig. 7, but with a new choke geometry, is also simulated and compared with the results of that with ordinary choke geometry. The absorption curves are plotted in Fig. 11, showing that the second fully reflected frequency is about $42 \mathrm{GHz}$ in the new choke.

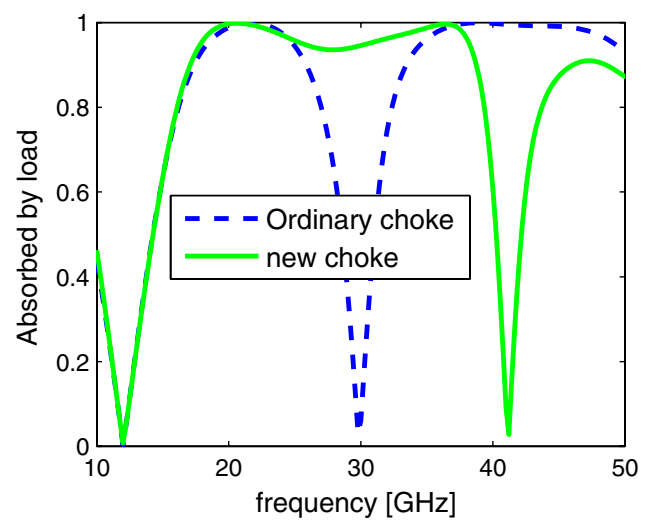

FIG. 11. Absorption curve of new choke (HFSS simulation). 
This feature can also be visualized on a Smith chart. In Fig. 12, the right-top subfigure shows the impedance of modes at the joint plane of two sections. The accelerating mode has inductive reactance while $29 \mathrm{GHz}$ has capacitive reactance. The impedance transformer at the joint plane of two sections multiplies them. The accelerating mode will rotate clockwise while $29 \mathrm{GHz}$ will rotate counterclockwise. At the termination of an ordinary choke, $29 \mathrm{GHz}$ is almost fully reflected so in a Smith chart it is close to the open point (shown in the left-bottom subfigure in Fig. 12). Given the effect from the two sections, at the termination of choke, the accelerating mode rotates forwards while the $29 \mathrm{GHz}$ mode rotates backwards. In order to tune the accelerating mode to the open point, smaller sizes of choke are used to make all modes rotate counterclockwise. The $29 \mathrm{GHz}$ mode will rotate further away from the open point. A higher frequency mode moves to this point. Consequently, the second fully reflected frequency is higher for the two-sections choke.

In order to reduce the reactance of higher order modes, section " $a$ " of the choke is designed to have a narrower gap than the inner radial line. Figure 1 shows this so-called "thin-neck choke." The joint plane of the choke and the radial line performs as another impedance transformer. So the impedance at the bottom of the choke and the radial line will be

$$
\begin{aligned}
& z_{3}=j a, \quad z_{2}^{\prime}=1+j \frac{a}{M}, \\
&|\Gamma|=\left|\frac{z_{2}^{\prime}-1}{z_{2}^{\prime}+1}\right|=\left|\frac{j a}{2 M+j a}\right|=\sqrt{\frac{a^{2}}{4 M^{2}+a^{2}}}
\end{aligned}
$$

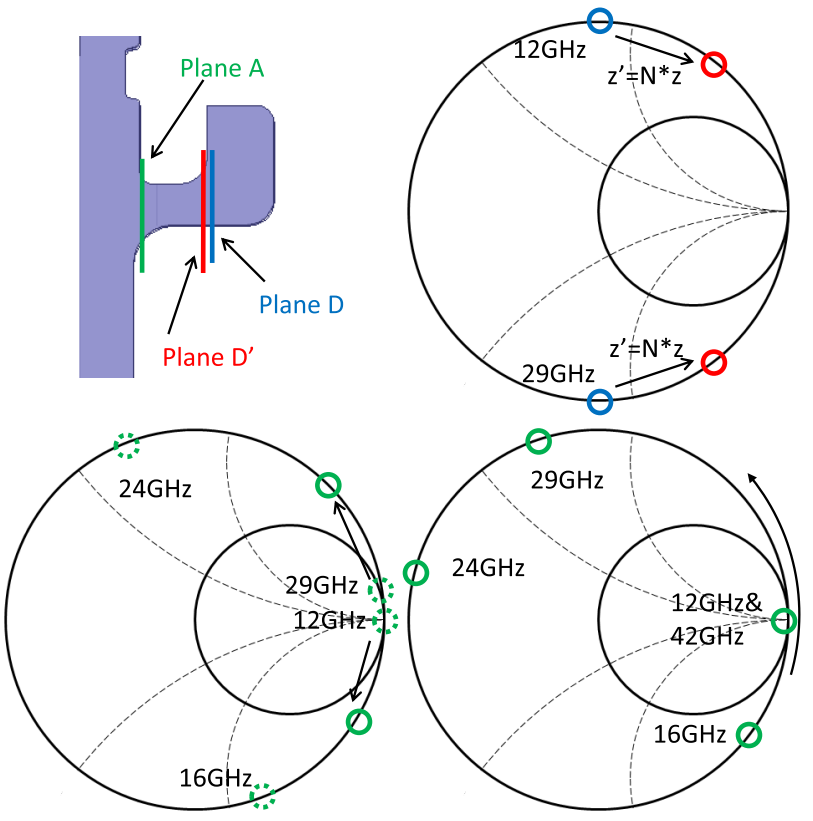

FIG. 12. Smith chart explanation for two sections choke: right top-impedance at junction of two sections; left bottom-impedance at termination of choke; right bottom-tune back $12 \mathrm{GHz}$.

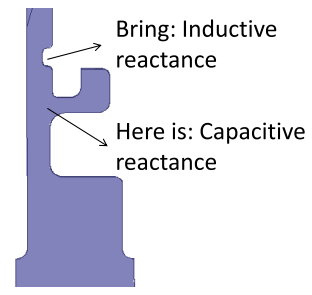

FIG. 13. Impedance match at outer radial line.

where $a$ is the reactance of higher order mode, $z_{3}$ is the impedance at the choke (reference plane A in Fig. 2), $z_{2}^{\prime}$ is the impedance at the joint plane of the choke, and the outer radial line (reference plane $\mathrm{T}$ in Fig. 2). $M$ indicates the ratio of the impedance transformer, and is the ratio of the gap of the radial line and the gap of the choke. $\Gamma$ is the reflection at choke. In the case of smaller gap of choke, $M$ is more than 1 . Consequently, the reactance of higher order modes will be reduced so the reflection will be smaller. However, the reactance of the accelerating mode is still infinite, then it is not affected by this thin-neck design.

Increasing this ratio $M$ will lead to a higher surface electrical field in the choke and in this way cause a reduction in high-gradient performance. Consequently, the range of ratio $M$ is limited. In the choke design shown in Fig. 9, the gaps of the radial line and the choke are set to 1.6 and $1.2 \mathrm{~mm}$.

Although the maximum surface field is on the iris, highpower tests in SLAC [12] have shown that choke-mode cells with $1 \mathrm{~mm}$ gap have a high breakdown rate at some points of the inner radial line, while the one with $4 \mathrm{~mm}$ gap had few breakdowns. Multipacting enhanced by a narrow gap is a possible reason of higher breakdown rate. Therefore high-power tests will be necessary to determine if the design shown in Fig. 9 is acceptable.

\section{B. Impedance matching element at outer radial line}

The first passband dipole modes near 16 and $18 \mathrm{GHz}$ in the choke-mode cavity have very high $(R / Q)_{\perp}$. But the "two sections" design decrease the absorption of these two modes. As shown in Fig. 12, the reason is that the capacitive reactance

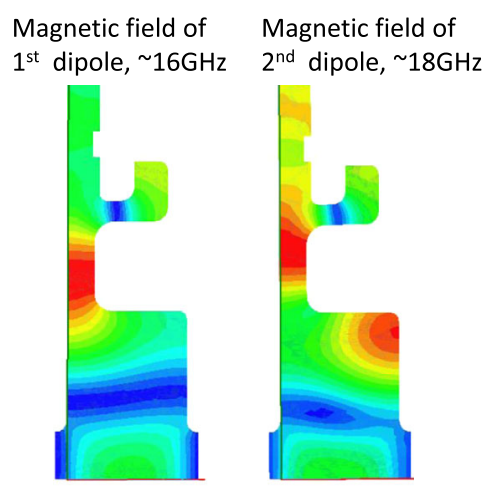

FIG. 14. Magnetic field of two dipole modes. 
TABLE II. Size change in each cell for detuning method to get $3 \mathrm{GHz}$ mode separation. $\Delta c_{1}$ indicates radius change of main cavity. $\Delta c_{2}$ indicates radius change of inner radial line.

\begin{tabular}{lcc}
\hline \hline Cells & $\Delta c_{1}$ & $\Delta c_{2}$ \\
\hline First cell (mm) & -0.243 & 1.0 \\
Middle cell (mm) & -0.161 & 0.7 \\
Last cell $(\mathrm{mm})$ & -0.087 & 0.4 \\
\hline \hline
\end{tabular}

of these two modes is higher so the reflection is increased. A new outer radial line with impedance match is designed to reduce this capacitive reactance (shown in Fig. 13). The step at the outer radial line provides capacitive reactance but at the junction point it turns to inductive reactance.

The impedance match method at the outer radial line may increase the reflection of a number of modes at the same time. However, since these modes do not have high $(R / Q)_{\perp}$, the damping effect is still better than the choke geometry without impedance match.

\section{Detuning method}

The two first-passband dipole modes near 16 and $18 \mathrm{GHz}$ have similar $(R / Q)_{\perp}$. If the phase difference between them is $180^{\circ}$ at $0.15 \mathrm{~m}$ (position of second bunch), they will cancel each other. This is taking place when the frequency separation is 1 or $3 \mathrm{GHz}$. The total transverse kick can be minimized if these two dipole modes have proper frequency separation and similar decay rate.

The frequency difference of the two dipole modes is about $2.5 \mathrm{GHz}$ for the new choke geometry with original cells. The magnetic fields of these two modes are shown in Fig. 14: the field is higher near the inner radial line for both modes. A change in the size of inner radial line would be a clear way to detune the frequency. In the choke geometry design shown in Fig. 9, the radius of the inner radial line is

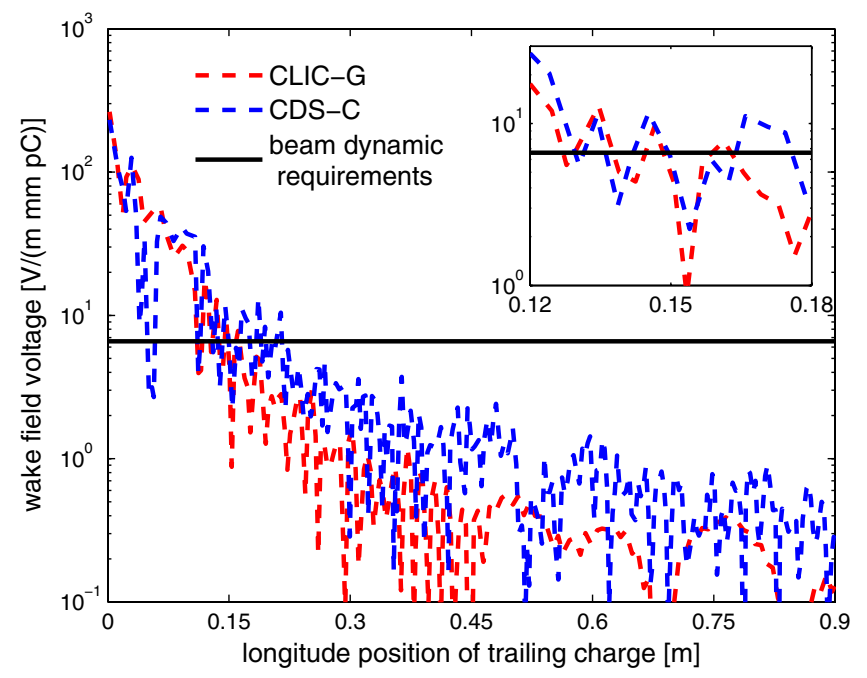

FIG. 15. Wakefield potential of CDS-C compared with CLIC-G.

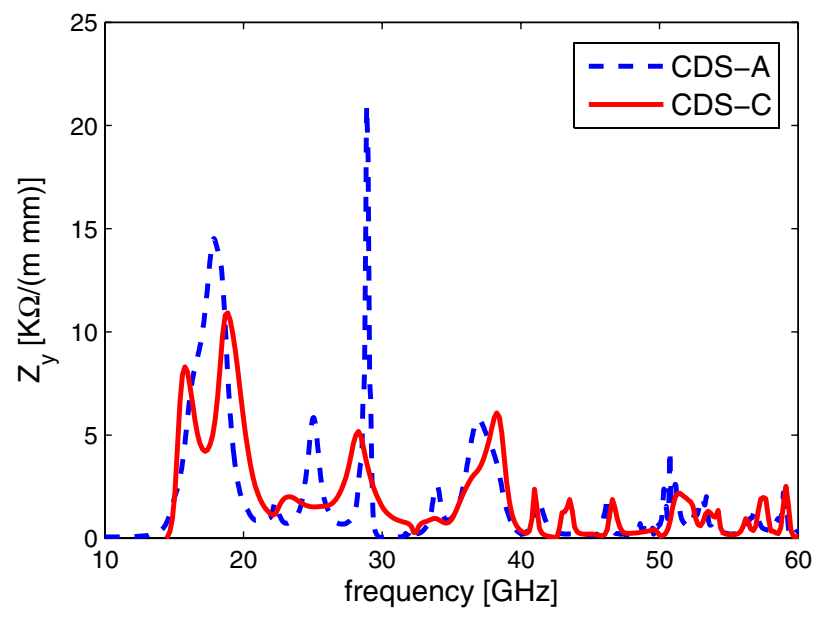

FIG. 16. Wakefield impedance spectrum of CDS-C compared with CDS-A.

increased to have a larger frequency separation. The radius of the main cavity is reduced in order to keep the frequency of the accelerating mode constant.

An optimization of the dimensions has been carried out and results are shown in Table II. The frequency difference is now $3 \mathrm{GHz}$ and the two dipole modes have a similar $Q$. The results will be shown in the following section.

\section{Wakefield damping results of new choke design}

With all three new features employed to minimize the transverse wake potential at the second bunch, we propose a structure design named "CDS-C." The wakefield potential simulated in GDFIDL is plotted in Fig. 15. The value at the second bunch is about $4 \mathrm{~V} /(\mathrm{m} \mathrm{mm} \mathrm{pC})$ at $0.15 \mathrm{~m}$ and meets beam dynamics requirements.

Figure 16 shows the wakefield impedance spectrum. Compared with the wakefield impedance of CDS-A, the high peak near $29 \mathrm{GHz}$ is much lower. Two peaks of lowpassband dipole modes are also lower, and the frequency difference between them is close to $3 \mathrm{GHz}$.

\section{RF PERFORMANCE OF CHOKE-MODE DAMPED STRUCTURE}

The CDS-C design still has the same iris dimensions as the CLIC-G nominal design. In Table III, the iris aperture and $\mathrm{rf}$ parameters of the first and last cell are listed and

TABLE III. The rf parameters of the first and the last cell of CDS-C and CLIC-G.

\begin{tabular}{lcc}
\hline \hline Parameters & CDS-C & CLIC-G \\
\hline Iris radius $(\mathrm{mm})$ & $3.15,2.35$ & $3.15,2.35$ \\
Iris thickness $(\mathrm{mm})$ & $1.67,1.00$ & $1.67,1.00$ \\
$Q$ factor $(\mathrm{Cu})$ & 4895,5385 & 5538,5738 \\
Shunt impedance $(\mathrm{M} \Omega / \mathrm{m})$ & 59,83 & 81,103 \\
Group velocity $(\% \mathrm{c})$ & $1.38,0.73$ & $1.65,0.83$ \\
\hline \hline
\end{tabular}




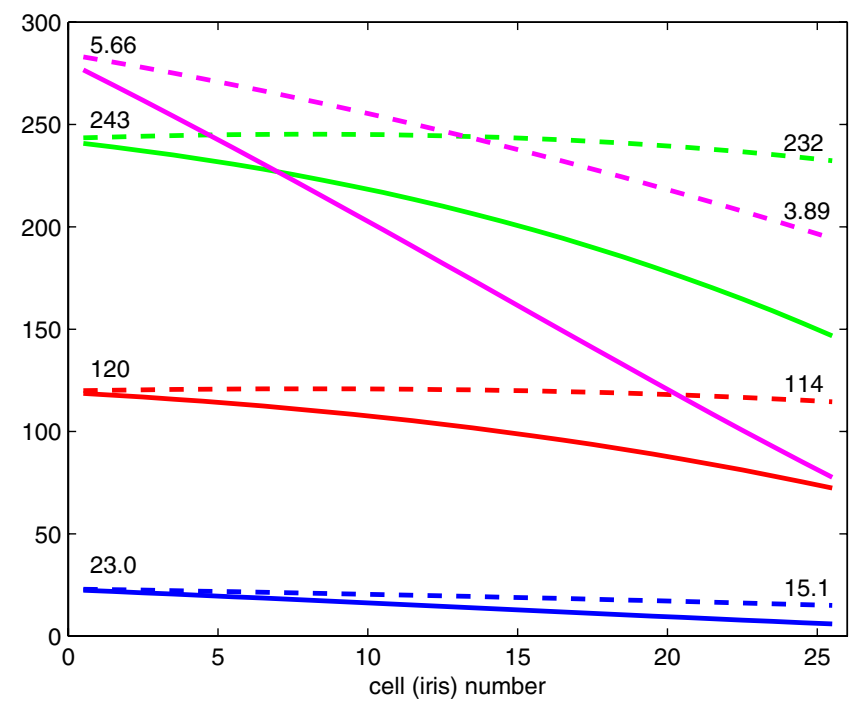

FIG. 17. The rf parameters of CDS-C: red is accelerating gradient $(\mathrm{MV} / \mathrm{m})$, green is maximum surface electrical field $(\mathrm{MV} / \mathrm{m})$, blue is pulsed temperature rise $(\mathrm{K})$, and magenta is 50 times maximum modified Poynting vector: $\mathrm{Sc} \times 50$ $\left(\mathrm{MW} / \mathrm{mm}^{2}\right)$ [13]. Dashed traces are unloaded and solid are beam loaded conditions.

compared with cells with waveguide damping in [1]. Cells with choke-mode damping have lower $Q$ and $R / Q$, which give lower rf-to-beam efficiency.

The whole structure has 24 regular cells and 2 matching cells. Thickness and radius of the iris of the cells are tapered linearly. In order to achieve the accelerating gradient of $100 \mathrm{MV} / \mathrm{m}$, a peak input power of $67.5 \mathrm{MW}$ is required. Distributions of rf parameters under this input power are shown in Fig. 17. Compared to the parameters of tapered structures with waveguide damping, listed in Table IV, the choke-mode damping structures needs more input power and the filling time is longer. But the pulsed temperature rise is much lower. The values in Table IV for CLIC-G are also calculated with 24 regular cells plus 2 matching cells by taken parameters from Ref. [1]. It is expected that a full optimization of the structure, taking advantage of the intrinsically lower $\delta T$, will restore some of the lost efficiency compared to CLIC-G.

TABLE IV. The rf parameters of CDS-C and CLIC-G.

\begin{tabular}{lcc}
\hline \hline Parameters & CDS-C & CLIC-G \\
\hline Max surface electric field & $246 \mathrm{MV} / \mathrm{m}$ & $235 \mathrm{MV} / \mathrm{m}$ \\
Peak input power & $67.5 \mathrm{MW}$ & $60.5 \mathrm{MW}$ \\
rf-to-beam efficiency & $23.7 \%$ & $27.5 \%$ \\
Maximum temperature rise & $22.9 \mathrm{~K}$ & $47.5 \mathrm{~K}$ \\
Maximum Sc & $5.66 \mathrm{MW} / \mathrm{mm}^{2}$ & $5.39 \mathrm{MW} / \mathrm{mm}^{2}$ \\
Filling time & $75.4 \mathrm{~ns}$ & $64.8 \mathrm{~ns}$ \\
\hline \hline
\end{tabular}

${ }^{\mathrm{a} S c}$ : modified Poynting vector [13].

\section{CONCLUSION}

A transmission line model for choke-mode structures is proposed to determine the principle of choke-mode damping and to aid the design process. The tuning formula is then used for simulation in the rf design of choke-mode cavity.

Guided by the transmission line model for choke mode, a new choke geometry with three new features is proposed. These improvements not only provide a better damping effect for almost all the dipole modes, but also use detuning to reduce the total transverse kick. The wakefield potential of the new design, CDS-C, with 24 tapered cells plus 2 matching cells, and this new choke scheme is below $6.6 \mathrm{~V} /(\mathrm{m} \mathrm{mm} \mathrm{pC})$ at the position of the second bunch, as required by the beam dynamics.

The rf parameters of the CDS-C structure are presented and compared with the nominal CLIC-G design with waveguide damping. The overall performance is promising. The choke-mode damping structures need more input power but have much lower pulsed temperature rise. A test prototype of CDS-C is designed and will be high power tested to compare with existing tests of T24 and TD24 series.

Future optimization of CDS series will include adjusting the iris aperture to have fast filling time and more bunch charge to increase the rf-to-beam efficiency. But since larger aperture means lower shunt impedance, there will be an optimization process.

\section{ACKNOWLEDGMENTS}

This work was supported by the National Natural Science Foundation of China (Grant No. 11135004).

[1] A. Grudiev and W. Wuensch, Proceedings of LINAC-10 (Jacow, Tsukuba, Japan, 2010) [http://accelconf.web.cern.ch/AccelConf/LINAC2010/papers/mop068.pdf].

[2] D. Schulte, in Proceedings of the 23rd Particle Accelerator Conference, Vancouver, Canada, 2009 (IEEE, Piscataway, NJ, 2009) [http://accelconf.web.cern.ch/AccelConf/PAC2009/ papers/fr5rfp055.pdf].

[3] T. Shintake, Jpn. J. Appl. Phys. 31, L1567 (1992).

[4] V. Khan. Ph.D thesis, University of Manchester, 2010.

[5] R. M. Jones, Phys. Rev. ST Accel. Beams 12, 104801 (2009).

[6] J. Shi, A. Grudiev, A. Olyunin, and W. Wuensch, in Proceedings of LINAC-10 (Jacow, Tsukuba, Japan, 2010) [http://accelconf.web.cern.ch/AccelConf/LINAC2010/ papers/mop022.pdf].

[7] J. Shi, H. Zha, A. Grudiev, and W. Wuensch, in Proceedings of IPAC-2011 (2011) [http://accelconf.web .cern.ch/AccelConf/IPAC2011/papers/mopc021.pdf].

[8] W. Bruns, http://www.gdfidl.de.

[9] N. Akasaka, T. Shintake, and H. Matsumoto, in Proceedings of LINAC-98 (Jacow, Victoria, BC, Canada, 
2008) [http://accelconf.web.cern.ch/AccelConf/198/PAPERS/ TU4080.PDF].

[10] Ansoft HFSS, www.ansoft.com.

[11] T. Shintake, in Proceedings of the Particle Accelerator Conference, Washington, DC, 1993 (IEEE, New York,
1993) [http://accelconf.web.cern.ch/AccelConf/p93/PDF/ PAC1993_1048.PDF].

[12] V. Dolgashev (private communication).

[13] A. Grudiev, S. Calatroni, and W. Wuensch, Phys. Rev. ST Accel. Beams 12, 102001 (2009). 